\title{
Evaluation of Task Shifting in Community-Based DOTS Program as an Effective Control Strategy for Tuberculosis
}

\author{
André P. Gabriel ${ }^{1}$ and Charles P. Mercado ${ }^{1,2}$ \\ ${ }^{1}$ Department of Medicine, Columbia University Medical Center, New York, \\ NY 10032, USA \\ ${ }^{2}$ Department of Biochemistry and Molecular Biology, University of Arkansas for \\ Medical Sciences, 4301 W. Markham Street, Slot 516, Little Rock, AR 72205, USA
}

Received 28 July 2011; Accepted 26 August 2011

Academic Editor: K. Kayser

Tuberculosis (TB) remains to be the most prevalent and debilitating pulmonary (PTB) infection in the world today, affecting about one-third of the world's population. TB is an endemic disease in many developing countries, and efforts at eliminating the disease remain futile. While the course of the disease is indolent with years of latency, the reactivation of the disease can pose serious pulmonary and systemic infections that compromise multiple organ functions which lead to respiratory failure or end-organ damage. Despite attempts to control and eradicate the mycobacterium, the prevalence of the disease remains high due to increasing population rate, persistence of poverty and poor health care, treatment failure, increasing multidrug resistance as a consequence of treatment failure and poor compliance, and existence of comorbid conditions that compromise immune response. Limited government resources to screen and monitor disease progression of TB in third world countries hamper the eradication of the disease. In response, we have evaluated the efficiency and effectivity of a Community-Based Directly Observed Treatment, Short-Course (CB-DOTS), which is an equally effective alternative strategy to health center DOTS.

KEYWORDS: Community based, directly observed treatment short course, tuberculosis control 


\section{INTRODUCTION}

Tuberculosis (TB) is the main microbial killer of adults in the world, killing over two million people per year and infecting over one-third of the world's population. It is a life-threatening infection that primarily affects the lungs. Currently, it is the main microbial killer of adults in the world, killing over two million people every year infecting over one-third of the world's population. Every second, a person is newly infected with the disease [1].

Primarily a respiratory disease, archaeological and genetic evidence indicates that most of the major forms of TB extant in humans today originated fifteen to twenty thousand years ago in Eastern Africa. As people migrated outward, TB did too. Archaeologists have found evidence of tuberculosis in millenia-old mummies from as far away as Egypt and Peru. Signs of skeletal TB (Pott's disease) were evident in Europe from Neolithic times (8000 BC), in ancient Egypt (1000 BC), and in the pre-Columbian New World [2]. TB was recognized as a contagious disease by the time of Hippocrates (400 BC), when it was termed "phthisis" (Greek from phthinein, to waste away) [3].

\section{NATURAL HISTORY OF THE DISEASE}

Mycobacterium tuberculosis, a tubercle bacillus, is the causative agent of TB. It belongs to a group of closely related organisms including $M$. africanum, M. bovis, and M. microti in the M. tuberculosis complex. It is a slow-growing, obligate aerobe and a facultative, intracellular parasite. The organism grows in parallel groups called cords. It retains many stains after decoloration with acid-alcohol, which is the basis of acidfast stains. Mycobacteria, such as M. tuberculosis, are aerobic, non-spore-forming, non-motile, facultative, intracellular, curved rods measuring $0.2-0.5 \mu \mathrm{m}$ by $2-4 \mu \mathrm{m}$. Their cell walls contain mycolic, acid-rich, long-chain glycolipids and phospholipoglycans (mycocides) that protect mycobacteria from cell lysosomal attack and also retain red basic fuchsin dye after acid rinsing (acid-fast stain) [4]. These pathogenic features of the TB bacilli make it resistant to cell death and thus necessitating the need for multiple-drug regimen.

\section{EPIDEMIOLOGICAL FEATURES OF TUBERCULOSIS}

\subsection{Route of Transmission}

Humans are the only known reservoir for M. tuberculosis. The organism is spread primarily as an airborne aerosol from infected to noninfected individuals (although transdermal and GI transmission has been reported). These droplets are $1-5 \mu \mathrm{m}$ in diameter; a single cough can generate 3000 infective droplets, with as few as 10 bacilli needed to initiate infection. When inhaled, droplet nuclei are deposited within the terminal airspaces of the lung. The organisms grow for 2-12 weeks until they reach 1000-10,000 in number, sufficient to elicit a cellular immune response that can be detected by a reaction to the tuberculin skin test. Because of the ability of $M$. tuberculosis to survive and proliferate within mononuclear phagocytes, which ingest the bacterium, it is able to invade local lymph nodes and spread to extrapulmonary sites, such as the bone marrow, liver, spleen, kidneys, bones, and brain, usually via hematogenous routes [5]. This multiorgan involvement necessitates longer treatment duration which consequently poses some issues with compliance to the treatment regimen.

The typical TB lesion is epithelioid granuloma with central caseation necrosis. The most common site of the primary lesion is within alveolar macrophages in subpleural regions of the lung. Bacilli proliferate locally and spread through the lymphatics to a hilar node, forming the Ghon complex. Initial lesions may heal and the infection, may become latent before symptomatic disease occurs. Smaller tubercles may resolve completely. Fibrosis occurs when hydrolytic enzymes dissolve tubercles, and larger lesions are surrounded by a fibrous capsule. Such fibrocaseous nodules usually contain viable mycobacteria and are potential lifelong foci for reactivation or cavitation. Some nodules calcify or ossify and are seen easily on chest radiographs [6].

If the host is unable to arrest the initial infection, the patient develops progressive, primary TB with tuberculous pneumonia in the lower and middle lobes of the lung. Purulent exudates with large numbers 
of acid-fast bacilli can be found in sputum and tissue. Subserosal granulomas may rupture into the pleural or pericardial spaces and create serous inflammation and effusions. These features make TB management extremely difficult as disease recurrence after a primary infection is likely.

\subsection{Risk Factors}

The four factors contributing to the increased risk of transmission are (1) number of organisms expelled, (2) concentration of organisms, (3) length of exposure time to contaminated air, and (4) immune status of the exposed individual [7].

Infected patients living in crowded or closed environments pose a particular risk to noninfected persons. Approximately $20 \%$ of people in household contact develop infection (positive tuberculin skin test). Microepidemics have occurred in closed environments such as submarines and on transcontinental flights. Populations at high risk for acquiring the infection also include hospital employees, inner-city residents, nursing home residents, and prisoners.

Increased risk of acquiring active disease occurs with HIV infection, intravenous (i.v.) drug abuse, alcoholism, diabetes mellitus (3-fold risk), silicosis, immunosuppressive therapy, cancer of the head and neck, hematologic malignancies, end-stage renal disease, intestinal bypass surgery or gastrectomy, chronic malabsorption syndromes, and low body weight. The risk is also higher in infants and young children less than five years of age [8].

Tumor necrosis factor-alpha (TNF- $\alpha$ ) antagonists, used in the treatment of rheumatoid arthritis, psoriasis, and several other autoimmune disorders, have been associated with a significantly increased risk for TB. Reports have included atypical presentations, extrapulmonary and disseminated disease, and deaths. Immunosuppressive therapy also includes chronic administration of systemic steroids and/or inhaled steroids [9].

Alterations in the host immune system that lead to decreased immune effectiveness can allow $M$. tuberculosis organisms to reactivate, with tubercular disease resulting from a combination of direct effects from the replicating infectious organism and from subsequent inappropriate host immune responses to tubercular antigens.

As an AIDS (acquired immune deficiency syndrome)-related opportunistic infection, TB is associated with HIV infections, with dual infections being frequently noted. Globally, coinfection with HIV is the highest in South Africa, India, and Nigeria. Persons with AIDS are 20-40 times more likely than immunocompetent persons to develop active TB. Correspondingly, TB is the leading cause of mortality among persons infected with HIV [10].

\subsection{Disease Burden}

Worldwide, TB is most common in Africa, the West Pacific, and Eastern Europe. These regions are plagued with factors that contribute to the spread of TB, including the presence of limited resources, HIV infection, and multidrug-resistant (MDR) TB. Consequently, although international public health efforts have put a huge curb on the rate of increase in TB, these regions account for the continued increase in global TB [11].

An estimated 9.27 million incident TB cases were reported internationally in 2007, an increase from 9.24 million in 2006. However, although the total number of cases increased, the number of cases per capita decreased from a global peak of 142 cases per 100,000 in 2004 to 139 cases per 100,000 in 2007. About 1.3 million deaths have been estimated among HIV-negative incident cases of TB (20 per 100,000 population) in the same year. An additional 456,000 deaths were among incident TB cases who were also HIV positive [12].

The high-TB-burden countries accounting for $80 \%$ of prevalent TB cases include Russia, India, Bangladesh, Pakistan, Indonesia, Philippines, Vietnam, Korea, China, Tibet, Hong Kong, Egypt, most SubSaharan African countries, Brazil, Mexico, Bolivia, Peru, Colombia, Dominican Republic, Ecuador, Puerto Rico, El Salvador, Nicaragua, Haiti, Honduras, and areas undergoing civil war [1]. 


\section{TASK SHIFTING IN COMMUNITY-BASED DIRECTLY OBSERVED TREATMENT, SHORT-COURSE (DOTS)}

Tuberculosis remains a public health problem of significance in the developing world, where already strained health service delivery systems continue to impede current control efforts. Globally, it is recognized that limited financial and health resources continue to strain efforts to control infectious diseases like TB, a condition worsened by the advent of HIV/AIDS.

The World Health Organization (WHO) and the International Union against TB and Lung Disease (IUATLD) promote universal adoption of Directly Observed Treatment, Short-Course (DOTS) for TB treatment as a means to improve TB treatment outcomes. The DOTS strategy focuses on five main points of action. These include government commitment to control TB, diagnosis based on sputum-smear microscopy tests done on patients who actively report TB symptoms, direct observation short-course chemotherapy treatments, a definite supply of drugs, and standardized reporting and recording of cases and treatment outcomes. The WHO advises that all TB patients should have at least the first two months of their therapy observed (and preferably the whole of it observed). This means an independent observer is tasked to watching patients swallow their anti-TB therapy. The DOTS treatment regimen is a multidrug combination of Isoniazid, Rifampicin, and Pyrazinamide in the first two months followed by Isoniazid and Rifampicin for the next six months. Because of these and the resource limitations of the health service sector in the developing world, there is a growing recognition of the need to decentralize TB control from hospitals and other primary care facilities to communities. One recommendation is to decentralize services in the communities through the task shifting framework. Task shifting calls for reallocating specific roles to health care workers who have had shorter training or lesser qualifications. In some cases, this includes delegating tasks to newly created cadres that have had specific competency-based training [13].

To understand the impact of task shifting in DOTS as an effective TB control strategy, a hypothetical TB Susceptible-Infectious-Recovered (SIR) Model is shown in Figure 1 [14]. This consists of four TB disease states: susceptible or uninfected (S), latently infected (L), infectious (I), and recovered persons (R). For TB, the infectious population is taken to include all individuals with active disease. Latently infected individuals are infected by the bacteria but do not have active disease and are therefore not contagious. Recovered individuals had active disease in the past but were able to contain the infection. Susceptible individuals have not had any infection yet.

In this model, the task shifting with the DOTS program aims to increase the rates of conversion of both infectious and noninfectious TB cases to the recovered group with aims of decreasing treatment failure rates. Treatment failure or inadequately treated TB cases, as discussed in several literatures, can potentially lead to multiple-drug-resistant TB strains [5]. Emergence of the MDR TB causes severe complications and requires a more intensive and therefore more expensive treatment regimens which causes severe burden on the already resource-limited developing countries.

For diseases such as TB, which require strict adherence to long-term daily therapy, it is particularly important that task shifting is implemented successfully. Evidence supporting showing task shifting or the modified Community-Based Directly Observed Treatment, Short-Course (CB-DOTS) as an effective TB control strategy is supported by several studies conducted in high TB burden.

One of these several studies was a qualitative study conducted by Mafigiri et al. in Kampala, Uganda comparing TB treatment success outcome categories (cured, treatment completion, death, treatment failure, defaulted and transferred out) in modified community-based DOTS versus clinic-based DOTS within the period of May 2005 and September 2006 [15]. In Uganda, the official CB-DOTS strategy involves the parish development committee, who are a small group chosen by the community to make decisions on social and economic development issues, including health matters. This group in liaison with the subcounty public health worker asks the community to nominate a volunteer who is willing and who is acceptable to the patient to deliver DOTS with overall supervision from the subcounty public health worker. Each participant selected a DOTS supporter from their social support network system. The primary study population consisted of new TB patients presenting to the clinic with their first episode of TB. Eligibility 


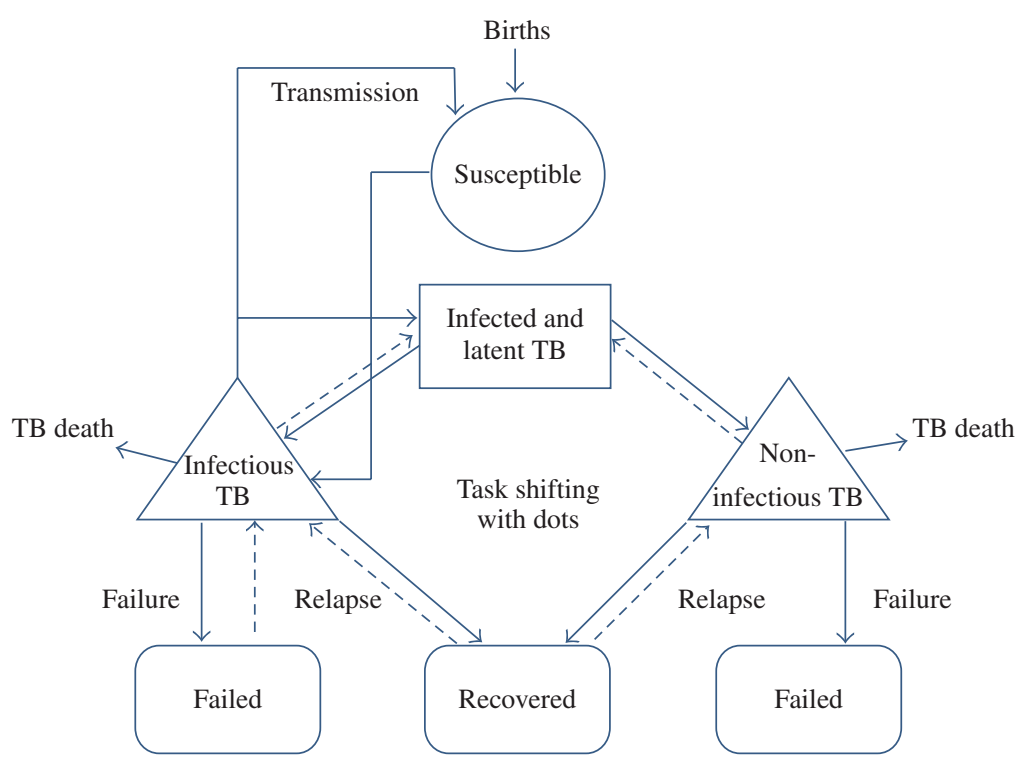

FIGURE 1: Schematic diagram for TB SILR Model. A hypothetical TB SIR Model consists of four TB disease states: susceptible or uninfected (S), latently infected (L), infectious (I), and recovered persons (R).

criteria include (1) sputum smear positive or smear negative with clinical signs of disease; (2) within one week of starting therapy; (3) no previous treatment for TB; (4) age 18 years and above; (5) resident in Kawempe Division in Kampala, Uganda. Each participant completed a baseline interview and was followed up on month two, five, and eight, coinciding with the recommended clinic visits for TB patients under DOTS. In total, 107 new smear-positive patients were recruited. Eighty-nine participants (83\%) selected the home-based DOTS strategy. Of the 89 patients who chose home-based DOTS, five $(6 \%)$ died, five $(6 \%)$ transferred care, eight $(9 \%)$ defaulted, and nine (10\%) were lost to follow up. Sixty-two patients (70\%) under home-based DOTS and 16 patients (89\%) under clinic-based DOTS had successful outcomes following complete tuberculosis therapy. Analysis of data showed no significant difference in the treatment rate between home-based DOTS and clinic-based DOTS (OR $=0.29$; 95\% CI: 0.06-1.34).

However, since this study design did not use randomization on treatment assignments, direct comparison between strategies is limited. There is a significant discordance between sample size of the home-based DOTS $(n=89)$ and that of clinic-based DOTS $(n=18)$. The sample size of the clinic-based DOTS is not large enough to observe the full range of outcomes as seen in the home-based group. Another limitation is that the patient tracking processes employed for the purpose of the research do not represent the standard clinical practice in this resource-limited setting. It is possible that, while this measure of care may have contributed to the high rate of acceptability and success of the modified CB-DOTS, it may not be sustainable in the current context of the national TB control efforts. Identifying Kawempe, the capital of Uganda and a relatively urban area, limits the generalizability of the results to other parts of the country where resources are more limited. Considering that the study was done in an urban setting, it would also be prudent to stratify the data obtained based on the socioeconomic status and educational level of the enrolled subjects.

To establish consistency of the association presented in Mafigiri et al.'s study in the rural setting, the study conducted by Adatu et al. is reviewed in this paper [16]. This prospective cohort study was done in Kiboga, a rural district in central Uganda, between 1995 and 1999. Both Kampala and Kiboga districts utilized the parish development committees and the subcounty health care workers for the implementation of the program. In this study design, the exposure is the CB-DOTS program with the TB treatment success being the outcome of interest. Treatment success is further categorized as (1) cured, (2) treatment completed, (3) treatment failure, (4) death, (5) treatment interruption, and (6) transfer. Eligible patients were identified 
as TB suspects meeting the diagnosis of active TB disease based on (1) positive smear and (2) negative smear but with clinical signs and symptoms and abnormal chest X-ray. Upon diagnosis of TB, patients are given the option to continue treatment with community-based DOTS or clinic-based DOTS. The study population consisted of 540 patients who underwent pre-CB-DOTS from 1995 to 1997 and 450 patients who were introduced to the CB-DOTS from 1998 to 1999. Following the introduction of CB-DOTS, there was a noted increase from $56 \%$ to $74 \%$ in the proportion of TB cases who were successfully treated (RR $=1.3,95 \% \mathrm{CI}: 1.2-1.5, P<0.001)$. The authors hypothesize that the rural setting made the selection of community volunteers easier because of the sense of community among its members. In addition, training of staff, volunteers, and the community resulted in increased awareness, decreased stigma, and a more educated population. Many persons became more motivated after seeing the initial success of CB-DOTS and witnessing cures among TB patients, which in turn simplified selection of community volunteers, training, treatment adherence and TB care.

Despite the encouraging results on treatment outcome among TB cases, data from 1997-1998 showed increased TB deaths even after the introduction of CB-DOTS (from 12.5\% in 1995 to 14.9 in 1998). Such a high death rate makes it difficult or impossible to achieve the $85 \%$ WHO target for treatment success. The factor most likely responsible for this high death rate is HIV coinfection which was not routinely tested for TB patients. Estimates, however, in the neighboring capital district of Kampala, showed that two-thirds of pulmonary TB cases were HIV seropositive.

\subsection{Sample Selection Bias}

The overall presentation of the results in both studies is fairly clear but the method used for assigning the sample population for either treatment arms increases the chance for sample selection bias. Since randomization was not used in the study design, patients enrolled in both studies were given an option to choose between community-based or clinic-based DOTS. Most of these patients chose to be in the CBDOTS. This led to an unequal distribution of samples to both treatment arms with the clinic-based DOTS sample group not having enough numbers to show all TB treatment outcomes compared to the CB-DOTS sample group. A bigger sample size for the clinic-based DOTS might report more favorable or superior TB treatment outcomes compared to CB-DOTS than what was originally observed (i.e., a lowered odds ratio in Mafigiri et al.'s study). Thus, the results would have been biased toward the null or show a weaker association than what was actually presented.

\subsection{Confounding}

There were a few variables associated with TB treatment outcomes that authors in both studies failed to address. These are age, socioeconomic status, neighborhood, BMI, education, history of head and neck cancer, use of immunosuppressive therapy, and most importantly HIV coinfection. To establish HIV coinfection as a potential confounder in both studies, however, is difficult considering that HIV status was not routinely done at baseline for TB diagnosis. We do, however, know that HIV, by causing a weakened immune system, increases the chances of acquiring contagious diseases including TB and indirectly increases the exposure of interest by seeking treatment of diagnosed TB cases with CBDOTS. Furthermore, weakened immune system secondary to HIV infection renders antimicrobial treatment difficult to completely eradicate the TB bacilli. This can lead to treatment failures and morbidities such as relapse or death. Both of which are the measured treatment outcomes in the two studies we have reviewed. Without any data on the HIV status entering both treatment arms, HIV coinfection cannot be fully established as a potential confounder.

\subsection{Assessment of Causality}

Evidence of association presented by Mafigiri et al. and Adatu et al.'s studies can be evaluated using Sir Austin Bradford Hill's criteria for causation [17]. 
Consistent with the study conducted by Newell et al. in Nepal, community-based DOTS is equally effective compared to clinic-based DOTS in high-burden countries [18].

In both studies, the sample population was managed through CB-DOTS (our exposure of interest), followed up time, and TB treatment success (categorized as cured, treatment completed, treatment failure, death, treatment interruption, and transfer) was measured as our outcome of interest. Since the measured outcome followed the exposure of interest, the associations presented in the studies meet the criteria for temporality.

The sample selection bias as mentioned previously is a major issue in both studies. Due to the skewed distribution of the sample population, with less subjects choosing the clinic-based DOTS, there is a chance that the measures of association presented may have been overestimated. Strength of association criterion, in this case, cannot be fully met by both studies. A more robust methodology and study design should be utilized in future trials.

\subsection{Generalizability}

In these two studies, both authors have recognized that a highly organized community structure such as that of the parish development committee and a well-established network of subcounty health care workers are the key factors in the success of task shifting in CB-DOTS. Results of both studies can be generalized only to populations with well-structured community organizations having an available network of trained health care workers.

\section{GAP IN KNOWLEDGE}

The equal efficacy of the CB-DOTS compared to clinic-based DOTS in high-burden countries has been fairly established in both studies reviewed in this paper. However, larger-scale and methodologically sound clinical trials may still be indicated not only in the previously studied populations but also in other highTB-burden countries as well. Detailed studies should be performed to compare the structure, process, and effectiveness of the CB-DOTS strategy in each of the 22 countries in order to identify the fundamental attributes of a successful and efficient system that will bring us closer to the goals of the WHO Stop TB Strategy.

Another challenge is learning how to structure a program that would integrate both CB-DOTS and home-based HIV treatment programs. Pilot studies like that of Miti et al. in Zambia have produced encouraging results [19]. Given that 13 of the high-TB-burden countries are found in Africa where HIV is also endemic and an association of HIV coinfection with low TB treatment success outcomes has been established, the need to conduct similar studies is highly warranted.

\section{CONCLUSION}

TB remains as a primary public-health concern in poor and developing countries. Current efforts which attempt at controlling the spread and treating the disease are strained and impeded by poor health service delivery system, lack of government resources, population explosion, and, recently, the emergence of multiple-drug-resistance TB strains. Globally, it is recognized that limited financial and health resources continue to strain efforts to control TB.

Task shifting with the DOTS program, which aims to increase the rates of conversion of both infectious and noninfectious TB cases to a recovered group, is an effective approach to monitor treatment, patient compliance, disease relapse, and prevention of emergence of MDR-TB. The DOTS strategy focuses on five main actions which are essential for the successful treatment and eradication of TB: (1) government commitment to control $\mathrm{TB}$, (2) diagnosis based on sputum-smear microscopy tests done on patients 
who actively report TB symptoms, (3) direct observation short-course chemotherapy treatments, (4) a definite supply of drugs, and (5) standardized reporting and recording of cases and treatment outcomes. In high-TB-burden countries, current evidence suggests that modified Community-Based Directly Observed Treatment, Short-Course (CB-DOTS) is as effective as the TB control strategy (DOTS).

While the implementation of these strategies remains a challenge in an attempt to eradicate TB, concerted efforts between the government, health care facilities, and local health care providers remain a cornerstone in delivering the basic necessities and tools in implementing the CB-DOTS program.

\section{REFERENCES}

[1] “WHO Tuberculosis Factsheet 2010/2011,” http://www.who.int/tb/publications/2010/factsheet_tb_2010_rev21feb 11.pdf.

[2] H. D. Chalke, "Some historical aspects of tuberculosis," Public Health, vol. 74, no. 3, pp. 83-95, 1959.

[3] T. M. Daniel, "The impact of tuberculosis on civilization," Infectious Disease Clinics of North America, vol. 18, no. 1, pp. 157-165, 2004.

[4] A. Konstantinos, “Testing for tuberculosis," Australian Prescriber, vol. 33, no. 1, pp. 12-18, 2010.

[5] Centers for Disease Control and Prevention (CDC), Division of Tuberculosis Elimination, Core Curriculum on Tuberculosis: What the Clinician Should Know, 4th edition, 2000.

[6] G. Assefa, Y. Nigussie, G. Aderaye, A. Worku, and L. Lindquist, "Chest X-ray evaluation of pneumonia-like syndromes in smear negative HIV-positive patients with atypical chest X-ray findings in Ethiopian setting," Ethiopian Medical Journal, vol. 49, no. 1, pp. 35-42, 2011.

[7] R. H. Pratt, C. A. Winston, J. S. Kammerer, and L. R. Armstrong, "Tuberculosis in older adults in the United States, 1993-2008," Journal of the American Geriatrics Society, vol. 59, no. 5, pp. 851-857, 2011.

[8] N. Shetty, M. Shemko, M. Vaz, and G. D'Souza, "An epidemiological evaluation of risk factors for tuberculosis in South India: a matched case control study," International Journal of Tuberculosis and Lung Disease, vol. 10, no. 1, pp. 80-86, 2006.

[9] M. Abebe, L. Kim, G. Rook et al., "Modulation of cell death by M. tuberculosis as a strategy for pathogen survival," Clinical and Developmental Immunology, vol. 2011, Article ID 678570, 11 pages, 2011.

[10] O. Koole, S. Thai, K. E. Khun et al., "Evaluation of the 2007 WHO guideline to improve the diagnosis of tuberculosis in ambulatory HIV-positive adults," PLoS One, vol. 6, no. 4, Article ID e18502, 2011.

[11] À. Orcau, J. A. Caylà, and J. A. Martínez, "Present epidemiology of tuberculosis. Prevention and control programs," Enfermedades Infecciosas y Microbiologia Clinica, vol. 29, supplement 1, pp. 2-7, 2011.

[12] H. J. Chum, R. J. O’Brien, T. M. Chonde, P. Graf, and H. L. Rieder, "An epidemiological study of tuberculosis and HIV infection in Tanzania, 1991-1993," AIDS, vol. 10, no. 3, pp. 299-309, 1996.

[13] "Treatment of Tuberculosis, American Thoracic Society, CDC and Infectious Diseases Society of America," American Journal of Respiratory and Critical Care Medicine, vol. 167, pp. 603-662, 2003.

[14] M. Casals, K. Guzmán, and J. A. Caylà, "Mathematical models used in the study of infectious diseases," Revista Espanola de Salud Publica, vol. 83, no. 5, pp. 689-695, 2009.

[15] D. K. Mafigiri, J. W. McGrath, and C. C. Whalen, "Task shifting for tuberculosis control: a qualitative study of community-based directly observed therapy in urban Uganda," Global Public Health. In press.

[16] F. Adatu, R. Odeke, M. Mugenyi et al., "Implementation of the DOTS strategy for tuberculosis control in rural Kiboga District, Uganda, offering patients the option of treatment supervision in the community, 1998-1999," International Journal of Tuberculosis and Lung Disease, vol. 7, no. 9, supplement 1, pp. S63-S71, 2003.

[17] A. Ascehngrau and G. R. Seage III, Essentials of Epidemiology in Public Health, Jones \& Bartlett Publishers, 2008.

[18] J. N. Newell, S. C. Baral, S. B. Pande, D. S. Bam, and P. Malla, "Family-member DOTS and community DOTS for tuberculosis control in Nepal: cluster-randomised controlled trial," The Lancet, vol. 367, no. 9514, pp. 903909, 2006. 
[19] S. Miti, V. Mfungwe, P. Reijer, and D. Maher, "Integration of tuberculosis treatment in a community-based home care programme for persons living with HIV/AIDS in Ndola, Zambia," International Journal of Tuberculosis and Lung Disease, vol. 7, no. 9, pp. S92-S98, 2003.

This article should be cited as follows:

André P. Gabriel and Charles P. Mercado, "Evaluation of Task Shifting in Community-Based DOTS Program as an Effective Control Strategy for Tuberculosis," TheScientificWorldJOURNAL, vol. 11, pp. 2178-2186, 2011. 


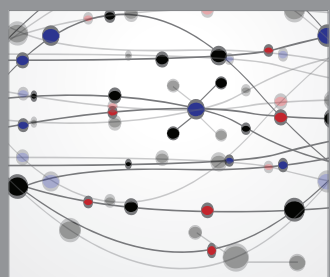

The Scientific World Journal
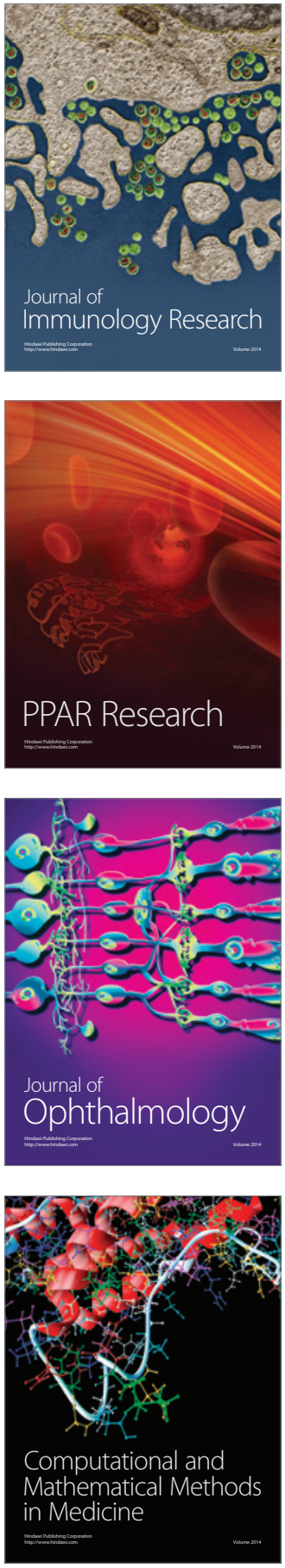

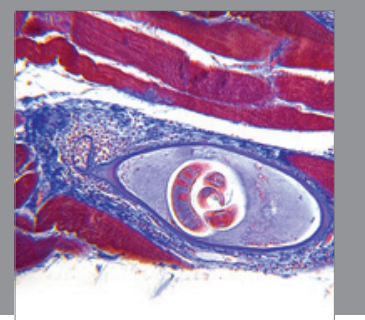

Gastroenterology

Research and Practice
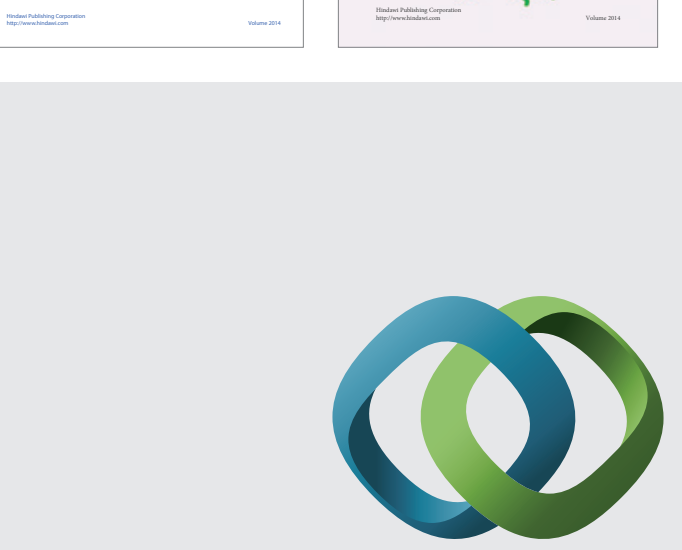

\section{Hindawi}

Submit your manuscripts at

http://www.hindawi.com
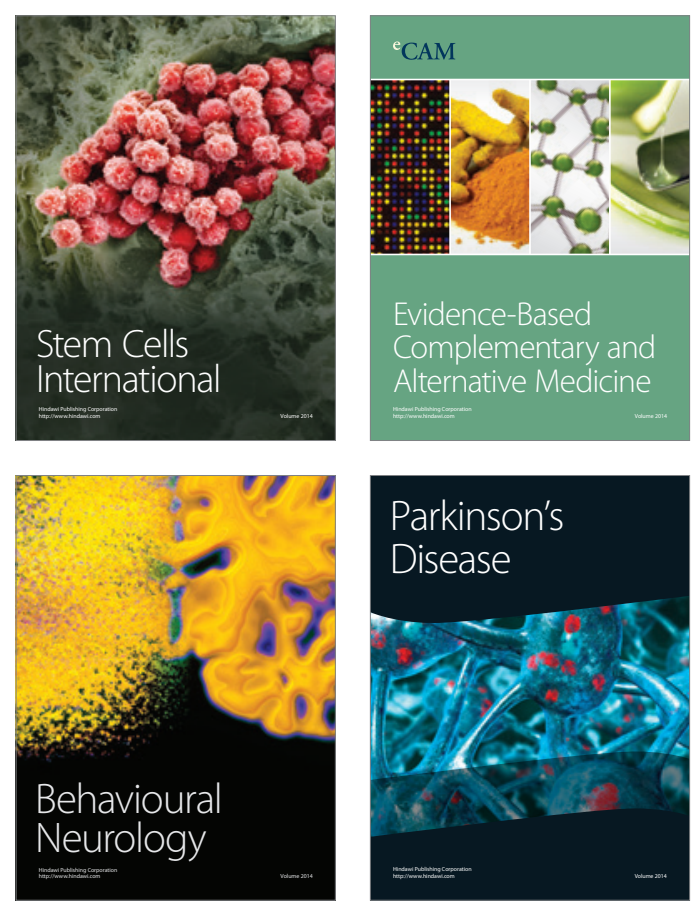

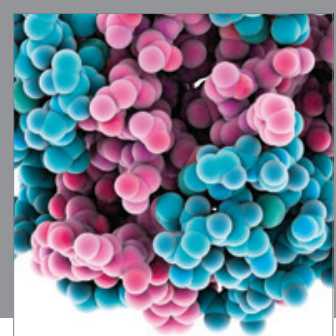

Journal of
Diabetes Research

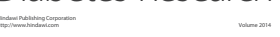

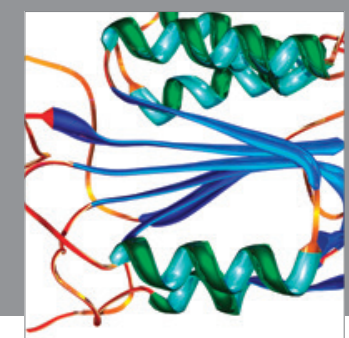

Disease Markers
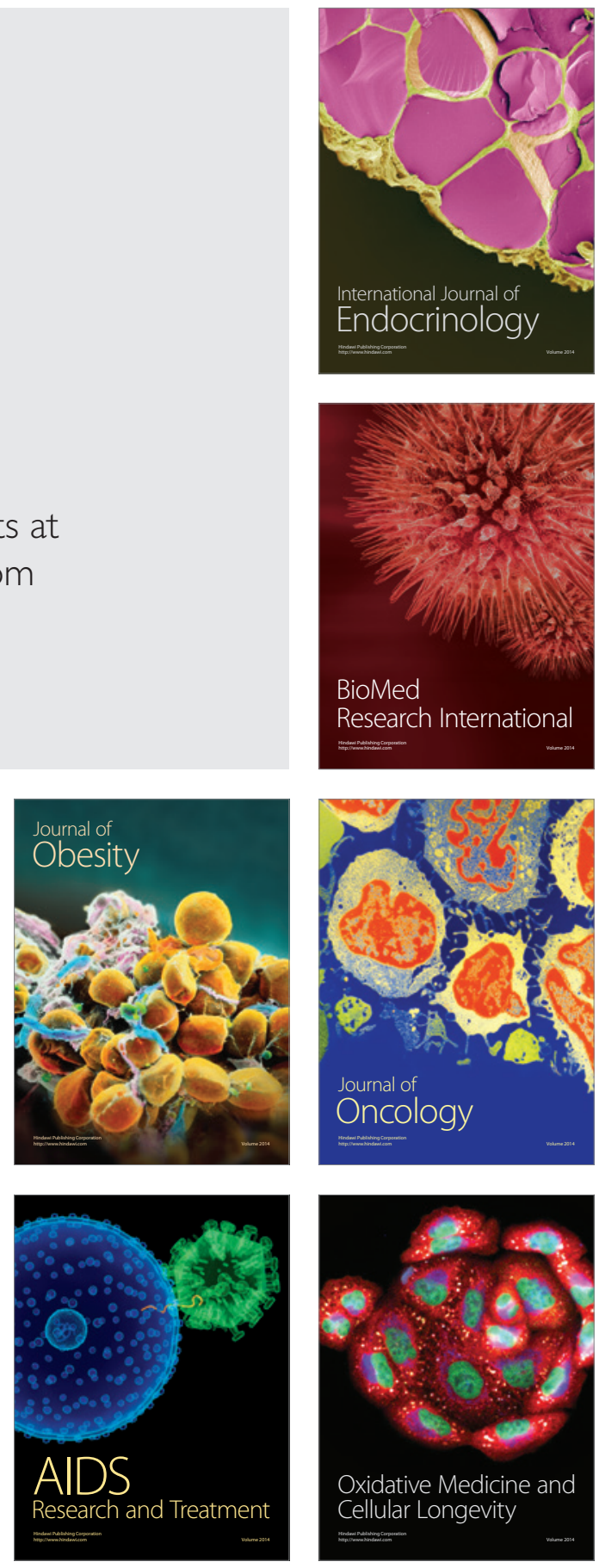